\title{
Detecção do trânsito planetário de um exoplaneta com um telescópio de pequena abertura
}

Detection of exoplanet transits using a small telescope

\author{
José Carlos Silva ${ }^{1}$, Artur Justiniano Roberto Junior*1], João Carlos Pereira Alves ${ }^{1}$ \\ ${ }^{1}$ Universidade Federal de Alfenas, Instituto de Ciências Exatas, Departamento de Física, Alfenas, MG, Brasil.
}

Recebido em 08 de abril de 2020. Revisado em 19 de maio de 2020. Aceito em 23 de maio de 2020.

\begin{abstract}
Neste trabalho mostramos como observar um trânsito planetário com um telescópio de pequena abertura, bem como a estratégia utilizada para selecionar o objeto, fazer as observações, o tratamento e a análise dos dados. Como exemplo observamos o trânsito planetário na estrela WASP 76 e fizemos uma estimativa do tempo do trânsito, do raio do planeta, do semieixo da órbita e da inclinação orbital. Os resultados obtidos mostram que mesmo com telescópios com configurações instrumentais modestas e instalados em locais com poluição luminosa é possível obter dados satisfatórios do trânsito planetário em estrelas brilhantes. A partir desse resultado defendemos que é possível realizar trabalhos de Astronomia observacional com telescópios de pequena abertura, como os inúmeros que existem nos diversos miniobservatórios instalados em universidades e centros de pesquisa do Brasil, de forma a proporcionar o letramento científico de estudantes de cursos de graduação e do ensino médio.

Palavras-chave: trânsito planetário, exoplaneta, curva de luz, miniobservatório, letramento científico.
\end{abstract}

In this paper we describe how to observe a planetary transit using a small aperture telescope, as well as the strategies used to select the object, make the observations, the treatment and the data analysis. As an example, we observed the planetary transit on the WASP 76 star and estimated the transit time, the radius, the orbit semi major-axis and the orbital inclination of the planet. The results show that telescopes with modest instrumental configuration can be used to obtain satisfactory data about exoplanets that transit bright stars. Based on this result, we propose that it is possible to do observational astronomy with small-aperture telescopes, such as the instruments that exist in the different small-observatories installed in universities and a research centers in Brazil, to provide scientific literacy for undergraduate students and from high school students.

Keywords: planetary transit, exoplanet, light curve, small-observatory, scientific literacy.

\section{Introdução}

Exoplanetas ou planetas extra-Solares são denominações atribuídas aos planetas que estão fora do Sistema Solar. O primeiro exoplaneta foi descoberto em 1992 orbitando o pulsar PSR1257+12 [1]. Já a primeira detecção de um exoplaneta orbitando uma estrela do tipo solar foi 51 Peg b em 1995 [2]. Em 2019 os autores dessa detecção, Mayor e Queloz, receberam o prêmio nobel de Física. Desde essa primeira medida, milhares de exoplanetas já foram descobertos, abrindo uma nova área de pesquisa na Astronomia.

Foram os telescópios espaciais CoRoT (Convection, Rotation and Transit) da Agência Espacial Europeia (ESA) e Kepler, da NASA, que impulsionaram as descobertas e os estudos dos exoplanetas [3, 4]. O CoRoT, lançado em 2006, foi a primeira missão espacial que possuía entre seus objetivos a pesquisa exoplanetária. Já a missão Kepler, lançada em 2009, foi a primeira dedicada exclusivamente à detecção de exoplanetas pela técnica de trânsito planetário (TTP), que será descrita em detalhes

*Endereço de correspondência: arturjustiniano@gmail.com no próximo capítulo. Essas missões descobriram milhares de exoplanetas [5] e ainda há muitos dados para serem analisados [6]. O que deve aumentar o número de exoplanetas detectados, principalmente similares ao planeta Terra.

Embora a missão Kepler tenha sido revolucionária ao descobrir que planetas do tamanho da Terra são comuns, a maior parte das estrelas no campo de Kepler fica a distâncias de centenas a milhares de parsecs (1pc equivale a 3,26 anos-luz), o que dificulta a obtenção de dados sobre esses planetas aqui da Terra.

Já o satélite TESS (Transiting Exoplanet Survey Satellite), lançado pela NASA em 2018 [7], foi projetado para examinar mais de $85 \%$ do céu (uma área 400 vezes maior que a coberta pelo Kepler) para procurar planetas em torno de estrelas próximas. As estrelas do TESS serão tipicamente 30 a 100 vezes mais brilhantes que as pesquisadas pelo satélite Kepler. Por isso, os planetas detectados em torno dessas estrelas terão medições muito mais refinadas da sua massa, do seu tamanho, da sua densidade e das propriedades atmosféricas.

Apesar dos telescópios espaciais serem a forma mais eficiente de detectar os exoplanetas, existem janelas de 
oportunidade para a investigação desses astros com os telescópios terrestres, como pode ser observado nas referências [8] e [9]. Além disso, devido ao avanço tecnológico dos detectores CCD's (Charge Coupled Device), cada vez mais eficientes e mais baratos, essa janela de oportunidades pode ser estendida para os telescópios de pequena abertura , como os das referências [10], [11] e [12], e aqueles instalados em miniobservatórios de diversas instituições de ensino e pesquisa do Brasil, como por exemplo as participantes do projeto Telescópios na Escola [13] .

Nesse artigo vamos mostrar como utilizar esses miniobservatórios para fazer observações de estrelas hospedeiras de exoplanetas e, a partir dos dados coletados, estimar alguns parâmetros físicos do sistema planetário. Trata-se de um trabalho que se propõe a contribuir com o desenvolvimento de trabalhos que envolvam a Astronomia observacional com alunos do ensino superior e até mesmo do ensino médio, de forma a aproximar as escolas públicas das instituições de ensino superior e contribuir para o letramento científico dos estudantes.

Para mostrar como detectar um exoplaneta em um miniobservatório apresentaremos os resultados das observações fotométricas do exoplaneta WASP-76 b, realizadas no miniobservatório astronômico da Unifal-MG. Primeiro será feita uma descrição do trânsito planetário e uma descrição teórica dos parâmetros físicos que podem ser estimados a partir da detecção do trânsito. Em seguida faremos a descrição da instrumentação utilizada, da estratégia escolhida para selecionar e fazer a observação da estrela, do procedimento utilizado no tratamento dos dados e, por fim, os resultados obtidos para o exoplaneta WASP-76 b. No Apêndice A apresentamos uma descrição, passo a passo, de como fazer a seleção dos objetos no site NASA Exoplanet Archieve [14]. No Apêndice B apresentamos uma descrição, passo a passo, de como utilizar o software AstroImageJ [15] para fazer o tratamento e a análise dos dados.

\section{O Trânsito Planetário}

O Trânsito Planetário (TP) é um fenômeno similar ao eclipse solar. Quando o planeta se desloca na frente do disco estelar ele bloqueia parte da radiação emitida pela estrela e o seu brilho é atenuado (Fig. 1). Esse deslocamento é denominado trânsito. Através do monitoramento do brilho da estrela ao longo do tempo é possível observar esse pequeno decréscimo. Chamamos de curva de luz o gráfico desse brilho aparente da estrela (fluxo) em função do tempo. A Técnica do Trânsito Planetário (TPP) consiste em observar esse trânsito, o que só é possível se houver um alinhamento da órbita do planeta com a nossa linha de visada.

A Figura 1 mostra as quatro fases principais do TP. Na fase 1 é observado o primeiro contato do exoplaneta com o disco estelar. Trata-se do início do trânsito, quando verificamos um decréscimo do fluxo na curva de luz. A fase 2 é o segundo contato, quando o exoplaneta está totalmente em frente ao disco estelar e constatamos a

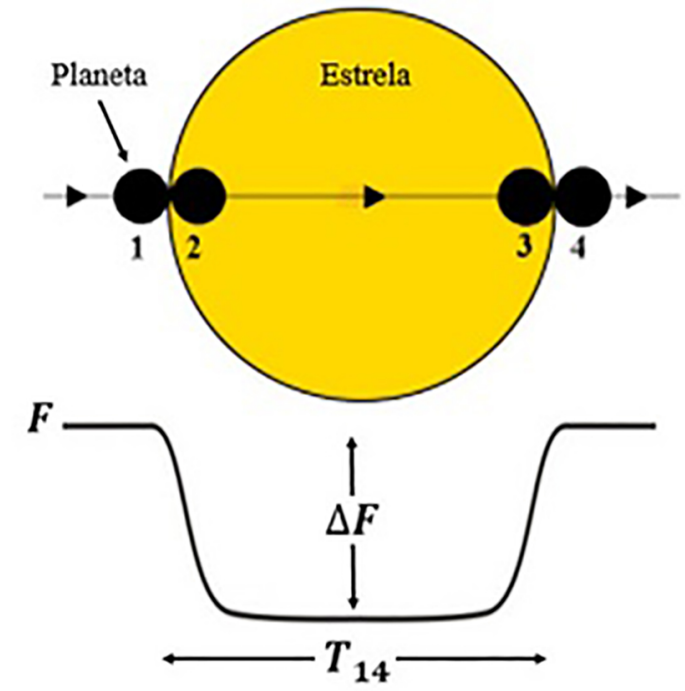

Figura 1: Curva de luz de uma estrela durante um trânsito planetário. A estrela é representada pela circunferência amarela e o planeta a preta. $F$ é o fluxo observado fora do trânsito, $\Delta F$ é a variação do fluxo e $\mathrm{T}_{14}$ o tempo do trânsito.

variação total do fluxo. $\mathrm{O}$ ponto entre o primeiro e o segundo contato é chamado de ingresso. Na fase 3 ocorre o terceiro contato, quando o exoplaneta começa a sair da frente do disco estelar e inicia o aumento do fluxo. Por fim, a fase 4, o quarto contato, quando o exoplaneta sai totalmente da frente do disco estelar, o egresso.

A possibilidade de descobrir exoplanetas similares a Júpiter por meio do TP foi prevista em 1952 [16] e em 2000 foi observado o primeiro TP [9] e em 2003 o exoplaneta OGLE-TR-56b foi o primeiro a ser detectado pela TTP [17]. A partir dessas detecções a TTP foi empregada em diversas missões [3, 4, e 7] e nos últimos anos passou a dominar o cenário das novas descobertas (Fig. 2).

\subsection{Parâmetros físicos}

Parâmetros orbitais do exoplaneta podem ser estimados a partir da análise da curva de luz da estrela hospedeira [19]. O raio do planeta $\left(R_{P}\right)$ pode ter seu valor estimado através da medida da variação do fluxo na curva de luz $\Delta F / F$, se o raio da estrela $\left(R_{*}\right)$ for conhecido. Neste caso, a relação entre essas grandezas pode ser escrita da seguinte forma:

$$
\left(\frac{R_{p}}{R_{*}}\right)^{2}=\frac{\Delta F}{F} .
$$

O leitor interessado em uma dedução detalhada da equação acima pode consultar a referência [20].

Além do raio do planeta, outros parâmetros orbitais do sistema planetário podem ser estimados a partir da análise da curva de luz gerada pelo TP [19]. A Figura 3 ilustra a órbita circular de um planeta ao redor de uma estrela. O TP é observado entre os pontos $A$ e $B$. 


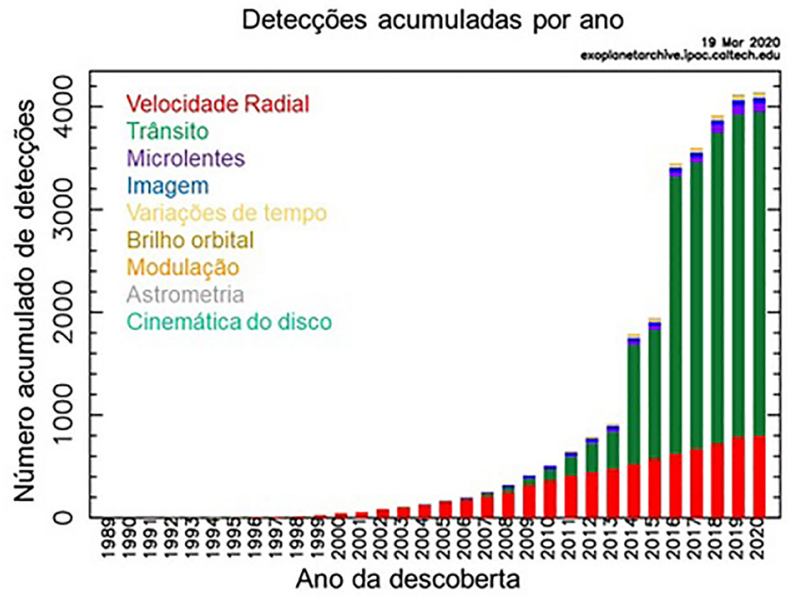

Figura 2: Número de planetas descobertos por diversas técnicas diferentes. A técnica de Trânsito Planetário é, atualmente, a de maior sucesso na descoberta de exoplanetas. Fonte: NASA Exoplanet Archieve [14].
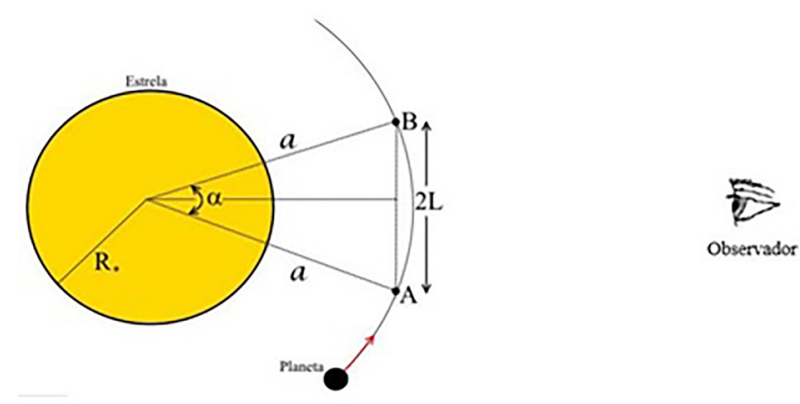

Figura 3: Projeção da visualização superior da órbita de um planeta. Onde $R_{*}$ é o raio da estrela, $a$ é o semieixo maior da órbita e o arco $A B \cong 2 \mathrm{~L}$. $L$ é a metade da trajetória percorrida pelo planeta, no disco da estrela, durante o trânsito.

Neste caso, considerando que a velocidade do planeta é constante, ela depende somente do raio da órbita $(a)$ e do período de translação $(P)$, ou seja:

$$
v=\frac{2 \pi a}{P}
$$

Se $T_{14}$ é o tempo necessário para o planeta percorrer o arco $A B$ e considerando que $v$ é constante, temos que:

$$
v=\frac{A B}{T_{14}}
$$

Igualando as Equações 2 e 3 e tomando $A B \cong 2 L$, temos que:

$$
T_{14}=\frac{P L}{\pi a} .
$$

Onde $L$ é a metade da trajetória percorrida pelo planeta, no disco da estrela, durante o trânsito. Utilizando a terceira Lei de Kepler e considerando que a massa da estrela é muito maior do que a massa do planeta $\left(M_{*} \gg\right.$ $\left.M_{p}\right)$ temos que:

$$
a^{3}=\frac{G M_{*}}{4 \pi^{2}} P^{2}
$$

Elevando a Equação 4 ao cubo e isolando $a^{3}$ e, em seguida, igualando com Equação 5, obtemos uma equação que relaciona a duração do trânsito $\left(T_{14}\right)$ com o parâmetro $L$.

$$
T_{14}^{3}=\frac{4 P}{\pi G M_{*}} L^{3}
$$

Onde $L$ é o termo que define se o planeta passa pelo equador, hemisfério norte ou sul da estrela. Pela Figura 4 podemos ver que

$$
L=\sqrt{\left(R_{*}+R p\right)^{2}-\left(R_{*} b\right)^{2}} .
$$

Onde o termo $R_{*} b$ representa um parâmetro que mede a distância da projeção da órbita do planeta em relação ao equador da estrela.

Observe que a duração do trânsito depende de $L$. Quando $R_{*} b=0 L$ tem seu maior valor e a duração do trânsito $\left(T_{14}\right)$ é máxima. Já quando $R_{*} b=R_{*}+R_{P}$ o parâmetro $L=0$ e nesse caso o trânsito não é observado.

A Figura 5 ilustra a posição da órbita do planeta em relação ao equador da estrela na perspectiva do observador. Essa posição é representada pelo termo $R_{*} b$ e $i$ é a

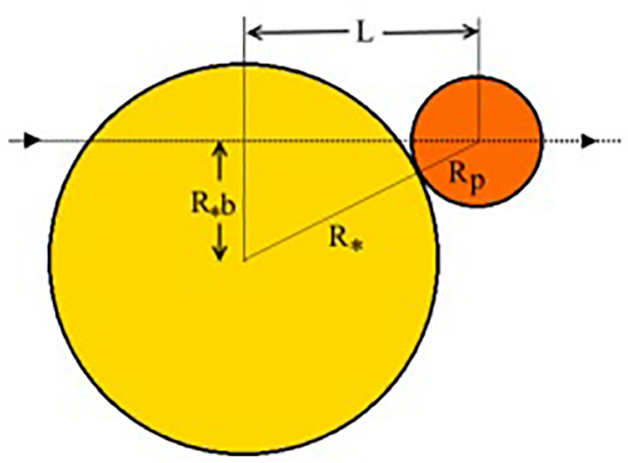

Figura 4: Representação geométrica do início de um trânsito planetário. Onde $R_{*}$ é o raio da estrela, $R_{p}$ é o raio do planeta, $R_{*} b$ marca posição da projeção da órbita do planeta em relação ao equador da estrela e $L$ é o parâmetro de impacto. Os raios da estrela e do planeta estão fora de escala.

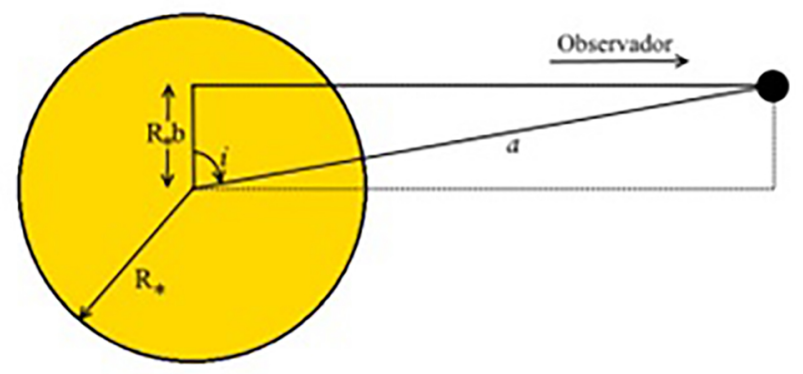

Figura 5: Representação geométrica da órbita da estrela. Onde $a$ é o semieixo maior da órbita, $i$ é a inclinação orbital do planeta, $R_{*}$ é o raio da estrela e $R_{*} b$ marca posição da órbita do planeta em relação ao equador da estrela. 
inclinação orbital do planeta em relação à linha de visada do observador. Matematicamente podemos escrever que

$$
R_{*} b=a \cos (i)
$$

Nesse capítulo mostramos que é possível obter da curva de luz a duração, $T_{14}$, e a profundidade, $\Delta F / F$, do trânsito. Desta última medida se obtém o raio do planeta $\left(R_{p}\right)$ pela Equação 1. Além disso, se a massa da estrela e o período orbital do planeta forem conhecidos é possível calcular o raio da órbita $(a)$, o parâmetro L e a inclinação orbital do planeta $(i)$. Tudo isso tendo como arcabouço teórico alguns conceitos de geometria e as leis de Kepler. Recomendamos ao leitor interessado em outros métodos de determinação dos parâmetros orbitais e da massa de exoplanetas a leitura da referência [22].

\section{Seleção de Objetos}

Para selecionar estrelas em sistemas exoplanetários que podem ser monitoradas por telescópios de pequena abertura e equipados com câmeras CCD's modestas, como os instalados em universidades e centros de divulgação científica, utilizamos o banco de dados NASA Exoplanet Archieve [14]. Este banco de dados combina informações de estrelas com exoplanetas confirmados a partir de dados de satélites como o CoRot, Kepler, TESS e também de dados de telescópios terrestres como os do projeto WASP (Wide Angle Search for Planets) [22]. A seleção das estrelas nesse banco de dados foi feita a partir dos seguintes critérios:

- trânsito planetário confirmado.

- Declinação da estrela $-60^{0} \leq \boldsymbol{\delta} \leq+30^{0}$.

- Magnitude aparente da estrela $\leq 10$.

- Tamanho do planeta $R_{P} \geq 0,1 R_{*}$.

Esses critérios foram escolhidos considerando a substancial poluição luminosa dos locais onde esses observatórios geralmente estão instalados e as suas posições geográficas. Optamos por selecionar objetos mais brilhantes para evitar longos tempos de integração, facilitar a identificação do alvo no campo e poder ser observado em noites cujas condições não estejam muito boas: céu relativamente ruim (nuvens), céu claro devido à Lua ou poluição luminosa. Além disso, para que o decréscimo de brilho da estrela durante o trânsito possa ser observado com telescópios de pequena abertura selecionamos apenas as estrelas que possuem planetas com raio superior a $10 \%$ do raio da estrela.

Como a possibilidade de observar um astro depende da latitude em que se encontra o telescópio, além da declinação do astro e da época do ano, a lista de objetos produzida neste trabalho não é universal. Procuramos selecionar objetos com uma boa distribuição em ascensão reta, de forma a permitir a realização de observação em qualquer época do ano, e também objetos que possam ser monitorados por observadores em todo o Brasil.

Com esses critérios foram selecionadas 6 estrelas em sistemas exoplanetários que podem ser monitoradas ao longo do ano. A Tabela 1 apresenta a lista das estrelas selecionadas, suas coordenadas equatoriais, magnitudes e raio. No Apêndice A disponibilizamos um tutorial de como obter essas informações no site da NASA, bem como identificar a melhor época do ano para observar o trânsito, conforme a localização do observatório. Neste trabalho, para demonstrar como obter uma curva de luz de um trânsito planetário escolhemos a estrela WASP-76.

\section{Observação e Tratamento dos Dados de WASP 76}

WASP-76 [23] é uma estrela do tipo solar com um raio de $1,73 R_{\text {Sol }}$, massa de $1,46 M_{\text {Sol }}$ e magnitude de 9,5. Essa estrela possui um único planeta detectado, WASP- 76b, com um período orbital de $P=1,81$ dias. Escolhemos essa estrela por ela ser brilhante e pelo fato de o trânsito poder ser observado em uma única noite de coleta de dados.

A efeméride para início da coleta de dados foi definida a partir da verificação, no site NASA Exoplanet Archive [14], da data em que um trânsito desse planeta poderia ser observado no local onde está o telescópio da UnifalMG. As informações de como obter essa efeméride para cada localidade estão no Apêndice A.

\subsection{Instrumentação utilizada}

Para monitorar WASP 76 utilizamos o miniobservatório da Universidade Federal de Alfenas (Unifal-MG). A instrumentação disponível (Fig. 6) é composta por um telescópio refletor do tipo Schmidt-Cassegrain da fabricante Celestron, modelo CGE Pro 1400, com um espelho primário esférico de $35,6 \mathrm{~cm}$, foco Schimidt-Cassegrain

Tabela 1: Dados das estrelas alvos selecionadas neste trabalho.

\begin{tabular}{|c|c|c|c|c|c|c|}
\hline \multicolumn{5}{|c|}{$\begin{array}{r}\text { Estrela } \\
\end{array}$} & \multicolumn{2}{|r|}{ Planeta } \\
\hline \multirow[t]{2}{*}{ Nome } & \multicolumn{2}{|c|}{ Coordenadas Equatoriais } & \multirow[t]{2}{*}{ Mag. } & \multirow[t]{2}{*}{ Raio (sol) } & \multirow[t]{2}{*}{ Nome } & \multirow[t]{2}{*}{ Período Orb (dias) } \\
\hline & $\alpha($ h m s) & $\delta(\mathrm{d} \mathrm{m} \mathrm{s})$ & & & & \\
\hline WASP-76 & 014631.86 & $+02 \quad 42 \quad 01.9$ & 9.5 & $1.73 \pm 0.04$ & WASP-76 b & 1.81 \\
\hline HR 858 & 025156.25 & -304852.3 & 6.4 & $1.31 \pm 0.01$ & HR 858 b & 3.58 \\
\hline KELT-19 A & 072602.28 & $+07 \quad 36 \quad 56.9$ & 9.8 & $1.83 \pm 0.01$ & KELT-19 A b & 4.61 \\
\hline WASP-69 & 210006.19 & -050539.9 & 9.9 & $0.86 \pm 0.03$ & WASP-69 b & 3.87 \\
\hline HD 209458 & 220310.77 & $+18 \quad 53 \quad 03.5$ & 7.6 & $1.19 \pm 0.02$ & HD 209458 b & 3.52 \\
\hline WASP-8 & 235936.07 & -350153.0 & 9.8 & $1.03 \pm 0.04$ & WASP-8 b & 8.15 \\
\hline
\end{tabular}




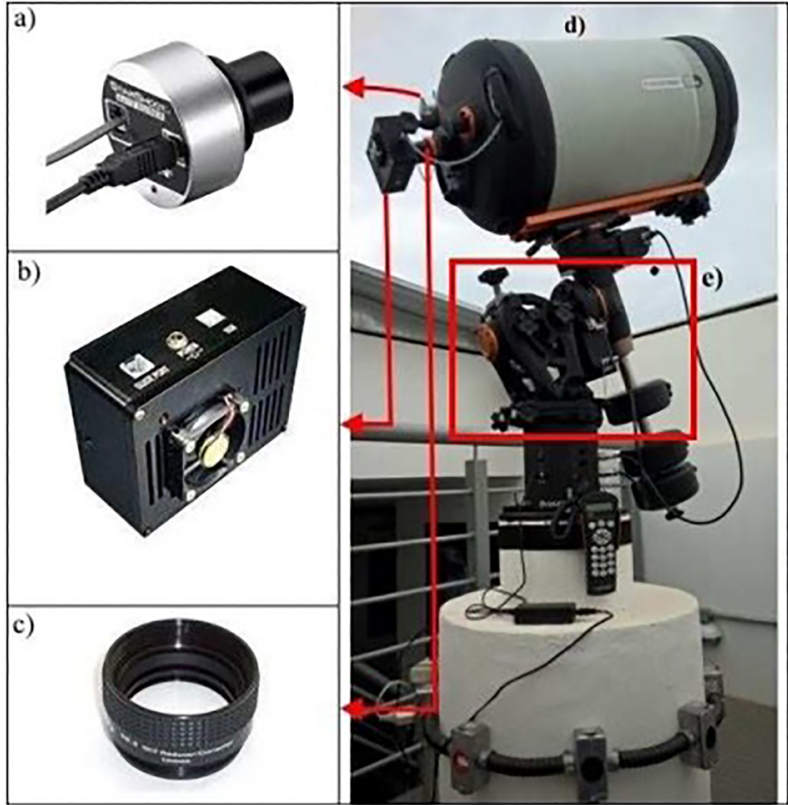

Figura 6: Instrumentação utilizada para observar WASP 76. a) Sensor de guiagem; b) Câmera CCD; c) Redutor focal; d) Telescópio Celestron, modelo CGE Pro 1400; e) Montagem equatorial alemã.

com espelho secundário hiperbólico convexo e uma placa corretora na abertura do tubo principal para corrigir as distorções ópticas provocadas pela esfericidade do espelho primário. A luz do astro é coletada no espelho primário e é refletida para o secundário, que, por sua vez, concentra a luz no plano focal do telescópio onde poderá encontrar uma ocular ou uma câmera CCD.

O telescópio repousa sobre uma montagem equatorial alemã automatizada que permite que o seu apontamento seja realizado de forma automática, via computador ou controle de mão, com um excelente retorno em termos de precisão quando associado ao sensor de guiagem. No plano focal está instalada uma câmera CCD, modelo $S T-402 M E$ com uma grade de $756 \times 510$ pixels com tamanho de 9 microns. A câmera possui um sistema de resfriamento de até $30^{\circ} \mathrm{C}$ abaixo da temperatura ambiente e seu nível de saturação é 65535 contagens por pixel. Combinando a câmera CCD e o telescópio, é possível obter um campo de visão $12,5 \times 12,5$ minutos de arco.

\subsection{Observação e tratamento dos dados}

Como o objetivo do trabalho foi observar um trânsito de WASP 76b optamos por começar a coletar os dados 1 hora antes do início previsto para ocorrer o trânsito, conforme as informações do site NASA Exoplanet Archieve [14] e fomos até 1 hora depois. Isso para garantir dados do ingresso do planeta em frente ao disco da estrela, bem como do seu egresso.

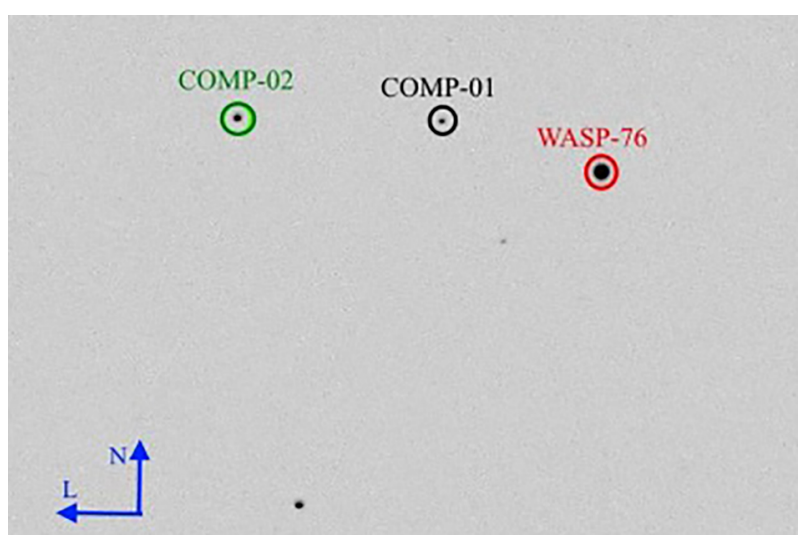

Figura 7: Imagem tratada do campo da estrela WASP -76 com as duas estrelas de comparação COMP - 01e COMP - 02 . As setas abaixo e a esquerda significam Leste (L) e Norte (N).

Apontamos o telescópio para o campo de WASP 76 e coletamos 1023 imagens ${ }^{1}$ sem filtro. Cada imagem foi feita com 20segundo de tempo de integração. Foram 6 horas seguidas de coleta de dados. A Figura 7 é uma imagem do campo da estrela WASP-76. São mostradas, além de WASP-76, as duas estrelas que foram utilizadas como comparação, $C O M P-01$ e $C O M P-02$. Estrelas de comparação são as que têm o brilho constante e estão no mesmo campo do objeto alvo.

Para o tratamento das imagens e extração dos fluxos das estrelas utilizamos o software livre AstroImageJ [15], administrado pela Universidade de Louisville. No Apêndice $\mathrm{B}$ disponibilizamos um roteiro detalhado, explicando passo a passo, como utilizar o AstroImageJ para fazer o tratamento das imagens, a extração dos fluxos, a obtenção da curva de luz e do valor medido do tempo do trânsito $\left(T_{14}\right)$ e do $\Delta F / F$ para a estimativa do raio do planeta $\left(R_{P}\right)$ e de outros parâmetros do sistema.

\section{Resultados}

Para construir a curva de luz de WASP-76 e das estrelas de comparação selecionamos as 570 melhores imagens e transferimos para o AstroImageJ. Fizemos o procedimento padrão para calibrar as imagens, minimizando os ruídos inseridos devido à temperatura da câmera CCD (corrente de escuro). Em seguida foram extraídos o fluxo de cada uma das estrelas de comparação e de WASP 76. O fluxo relativo de WASP 76 é a razão entre o fluxo dessa estrela e soma dos fluxos das duas estrelas de comparação [WASP76/(COMP-01+COMP - 02)]. O fluxo relativo da estrela de comparação 1 é a razão entre os fluxos $(C O M P-01 / C O M P-02)$ e o fluxo relativo da estrela de comparação 2 é a razão entre os fluxos (COMP $-02 / C O M P-01)$.

A Figura 8 mostra a curva de luz do trânsito planetário na estrela WASP 76. O planeta que produz esse

${ }^{1} \mathrm{O}$ leitor pode solicitar as imagens de WASP 76 ao autor principal pelo e-mail: jcarlos1905@gmail.com. 


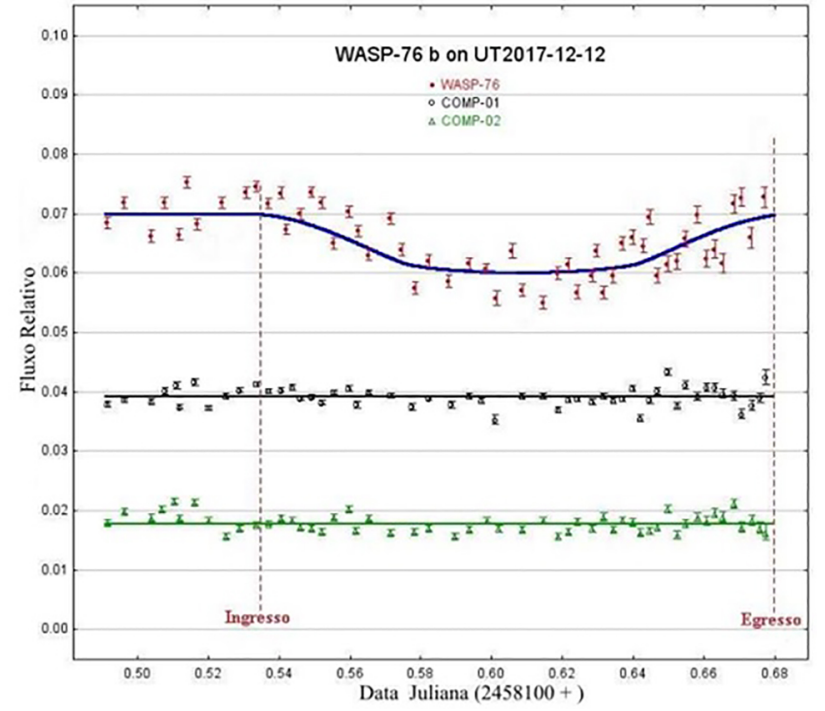

Figura 8: Curva de luz de WASP 76 e das estrelas de comparação. As linhas pontilhadas verticais marcam o início e o fim do trânsito planetário na estrela WASP 76. A linha azul é a curva de luz, modelada pelo AstrolmageJ, que melhor se ajusta aos dados.

decréscimo de brilho é denominado WASP 76b. Note que as duas estrelas de comparação possuem brilho constante, como era esperado. Superposta à curva de luz do trânsito, na cor azul, está curva de luz modelada pelo AstroImageJ e que melhor se ajusta aos dados. A estimativa de alguns parâmetros físicos do sistema (Fig. 9) é obtida a partir desse modelo. O leitor interessado poderá consultar as referências [24] e [25] para maiores detalhes sobre como o AstroImageJ faz esse modelo de curva de luz do trânsito planetário e como são obtidos os valores dos parâmetros. Aqui é importante esclarecer que o software não faz estimativas da precisão dos valores dos parâmetros. Ele apenas calcula o RMS e o $c h i^{2}$ do ajuste do modelo de curva de luz aos dados.

Para obter a duração do trânsito foi marcado na curva de luz o momento em que o planeta começa a passar pelo disco da estrela (ingresso) e o momento que ele deixa o disco (egresso). Esse intervalo é o tempo do trânsito, $T_{14}=0,1416$ dias. Além disso, o AstroImageJ obtém o valor do $\Delta F / F=0,0116$, a partir da medida da variação do fluxo de WASP 76 observado na curva de luz, e faz uma estimativa para o raio do planeta, $R_{p}=1,81 R_{J}$, com a Equação 1.

Para estimar o valor do raio da órbita, $a=0,033 \mathrm{AU}$ o AstroImageJ usa a Equação 5, terceira lei de Kepler, uma vez que o Período orbital $(\mathrm{P})$ do planeta é um parâmetro de entrada e a massa da estrela é obtida a partir de outro parâmetro de entrada, o raio da estrela. Com as Equações 4 e 5 foram calculados os valores de $L=1,066 R_{*}$ e $b=0$, 301.Já a inclinação orbital do planeta WASP $76 \mathrm{~b}$ em relação a nossa linha de visada, $i=86,0^{\circ}$, foi obtida com a Equação 8.

Ao compararmos (Tabela 2) os valores obtidos aqui com os que foram publicados em uma revista especializada [26] observamos que os nossos resultados são bastante satisfatórios em termos de aproximação com diferenças inferiores a $10 \%$ em relação aos encontrados em [26]. Apesar do objetivo principal do trabalho não ser a obtenção de estimativas refinadas para parâmetros físicos e geométricos desse sistema planetário e os resultados de [26] terem sido alcançados com equipamentos muito melhores e técnicas estatísticas sofisticadas para

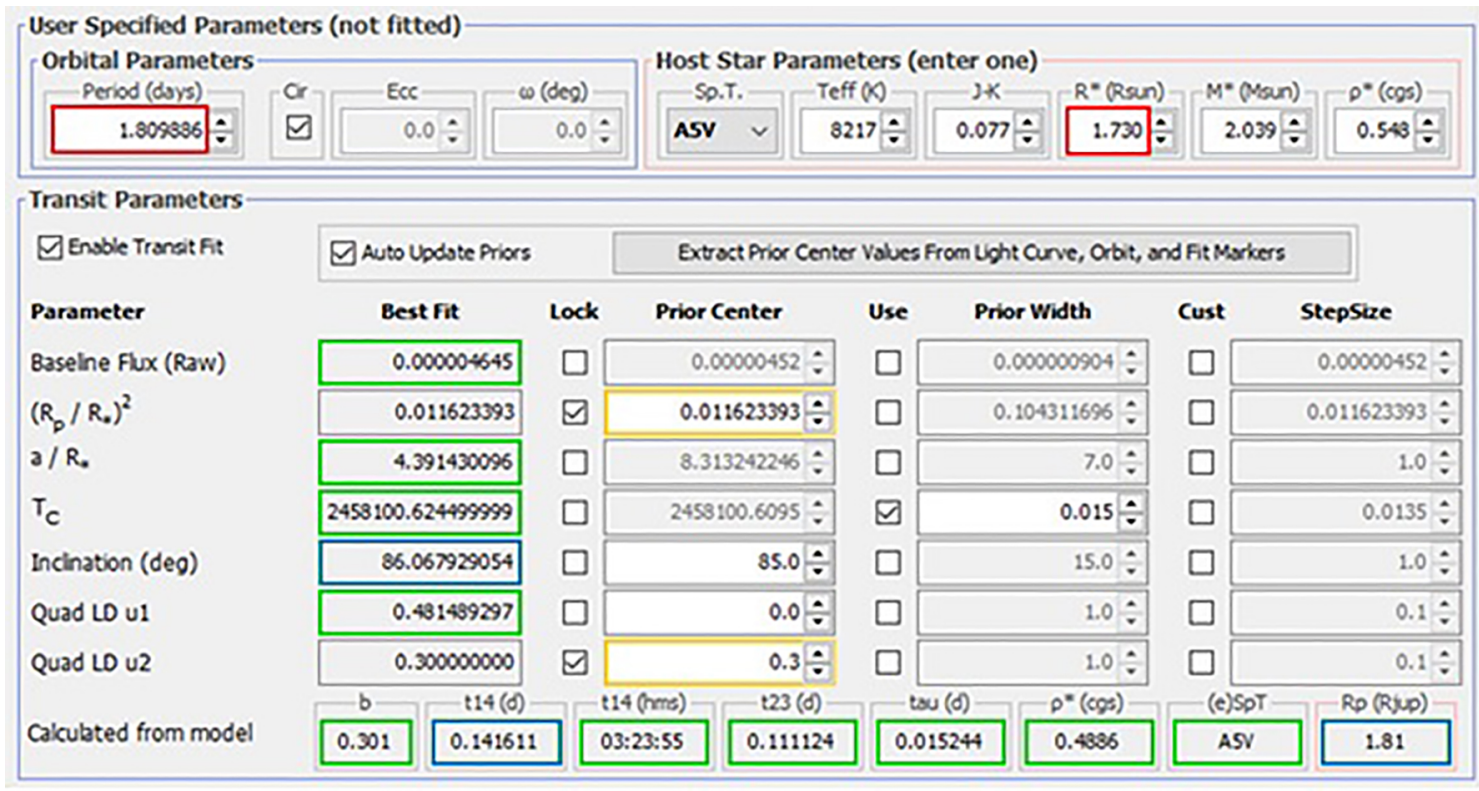

Figura 9: Tela principal do AstrolmageJ mostrando os parâmetros de entrada, o Período orbital do planeta $(P)$ e o Raio da estrela $R_{*}$ (retângulos vermelhos), e os parâmetros calculados pelo software, retângulos verdes. Destacamos na cor azul a inclinação orbital do planeta $(i)$, o tempo do trânsito $\left(T_{14}\right)$ e o raio do planeta $\left(R_{p}\right)$. 
Tabela 2: Comparação dos parâmetros de WASP 76b obtidos neste trabalho com os da literatura.

\begin{tabular}{lcc}
\hline Parâmetros & West e colaboradores [26] & Neste trabalho \\
\hline$T_{14}(d)$ & 0,1539 & 0,1416 \\
$R_{P}\left(R_{J}\right)$ & 1,83 & 1,81 \\
$a(U A)$ & 0,033 & 0,033 \\
$i($ graus $)$ & 88,6 & 86,0 \\
\hline
\end{tabular}

tratar e analisar os dados. Isto nos mostra que a proposta de utilizar miniobservatórios para observar o trânsito de planetas do tipo gigantes gasosos é viável.

\section{Consideração Finais}

Nesse trabalho buscamos demostrar que é possível observar um trânsito planetário com um telescópio de pequena abertura, semelhante aos inúmeros espalhados pelas universidades do Brasil, desde que a estrela seja brilhante e que o planeta seja do tipo gigante gasoso. Além disso, mostramos que é possível fazer boas estimativas de alguns parâmetros físicos do planeta.

A partir dos resultados alcançados defendemos que é possível desenvolver trabalhos que privilegiam a abordagem observacional da Astronomia tanto com alunos dos cursos de graduação quanto com professores e alunos de escolas públicas, de forma a aproximá-los das Instituições de Ensino Superior e as Instituições Científicas, Tecnológicas e de Inovação (ICT) que possuem telescópios. Essa aproximação é importante para a disseminação dos métodos científicos e para despertar a vocação dos alunos e professores da educação básica para as carreiras científicas. Além disso, a Astronomia, por ser uma ciência integradora de disciplinas como a Computação, a Matemática e a Física, pode ser usada para privilegiar o letramento científico e o uso de abordagens investigativas e de metodologias ativas de ensino, tanto na educação básica quanto superior.

\section{Material Suplementar}

O seguinte material suplementar está disponível online:

No Apêndice A disponibilizamos um material para auxiliar na seleção das estrelas e do melhor momento para a observação do trânsito.

No Apêndice B um material para auxiliar no tratamento e na análise dos dados com o software AstroImageJ.

\section{Referências}

[1] A. Wolszczan e D.A. Frail, Nature 355, 145 (1992).

[2] M. Mayor e D. Queloz, Nature 378, 355 (1995).

[3] Agência Espacial Europeia, Missão CoRoT, disponível em http://sci.esa.int/corot/, acessado em 15/05/2020.

[4] NASA, Missão Kepler, disponível em https://www.nasa. gov/mission_pages/kepler/overview/index.html acessado em 30/03/2020.
[5] NASA Exoplanet Archieve, disponível em https:// exoplanetarchive.ipac.caltech.edu/, acessado em $15 / 05 / 2020$.

[6] D.A. Fischer, A.W. Howard, G.P. Laughlin, B. Macintosh, S. Mahadevan, J. Sahlmann e J.C. Yee, arXiv:1505.06869 (2015).

[7] NASA, TESS Exoplanet Mission, disponível em https://www .nasa.gov/tess-transiting-exoplanetsurvey-satellite acessado em 15/05/2020.

[8] G.W. Henry, G.W. Marcy, P.P. Butler e S.S. Vogt, The Astrophysical Journal 529, L41 (2000).

[9] D. Charbonneau, T.M. Brown, D.W. Latham e M. Mayor, The Astrophysical Journal 529, L45 (2000).

[10] V.P. Hentunen, M. Nissinen, H. Haukka e H. Aartolahti, European Planetary Science Congress, disponível em https://meetingorganizer.copernicus.org/ EPSC2009/EPSC2009-119.pdf, acessado em 15/05/2020.

[11] D. Sergison, The Journal of the British Astronomical Association 123, 3 (2013).

[12] Amateur Detects Exoplanet Transit, disponível em https://skyandtelescope.org/astronomy-news/ amateur-detects-exoplanet-transit/, acessado em $15 / 05 / 2020$

[13] Telescópios na Escola, disponível em http: //www.telescopiosnaescola.pro.br/, acessado em 15/05/2020.

[14] NASA Exoplanet Archive, disponível em https: //exoplanetarchive.ipac.caltech.edu/index.html acessado em 15/05/2020.

[15] AstroImageJ, disponível em https://www.astro. louisville.edu/software/astroimagej/, acessado em $30 / 03 / 2020$

[16] O. Struve, The Observatory 72, 199 (1952).

[17] M. Konacki, G. Torres, S. Jha e D.D. Sasselov, Nature 421, 507 (2003).

[18] E. Martioli, Exoplanetas: O que são e como detectá-los. Dissertação de Mestrado, Instituto Nacional de Pesquisas Espaciais, São José dos Campos (2006).

[19] C.A. Haswell, Transiting Exoplanets: Measuring the Properties of Planetary Systems (Cambridge University Press, New York, 2010), v. 1.

[20] W.C. Santos e R.G.G. Amorim, Rev. Bras. Ens. Fís. 39, e2308 (2017).

[21] R.G.G. Amorim e W.C. Santos, Rev. Bras. Ens. Fís. 39, e1310 (2017).

[22] Wide Angle Search for Planets, disponível em https: //wasp-planets.net/, acessado 30/03/2020.

[23] WASP 76, disponível em http://exoplanet.eu/ catalog/wasp-76_b/, acessado em 30/03/2020.

[24] K.A. Collins, J.F. Kielkopf, K.G. Stassun e F.V. Hessman, arXiv:170104817v1 (2017).

[25] K. Mandel e E. Agol, The Astrophysical Journal 580, L171 (2002).

[26] R.G. West, C. Hellier, J.M. Almenara, D.R. Anderson, S.C.C. Barros, F. Bouchy, D.J.A. Brown, A. Collier Cameron, M. Deleuil, L. Delrez et al., Astronomy \& Astrophysics 585, A126 (2016). 